\title{
Measurement of the Double Longitudinal Spin Asymmetry in Inclusive Jet Production in Polarized p-p Collisions at $\sqrt{s}=200 \mathrm{GeV}$ at STAR
}

\author{
David Relyea (for the STAR Collaboration) \\ California Institute of Technology \\ 1200 E. California Blvd., Pasadena, CA 91125, USA
}

\begin{abstract}
One of the primary goals of the STAR spin program at RHIC is the measurement of the double longitudinal asymmetry $A_{L L}$ in inclusive jet production. This measurement will allow STAR to determine the polarized gluon distribution function $\Delta \mathrm{G}$ in the Bjorken $\mathrm{x}$ region of $0.03<\mathrm{x}<0.3$. Inclusive jet production is a theoretically desirable way to measure $\Delta \mathrm{G}$, as it is independent of fragmentation functions. This contribution presents STAR's preliminary 2005 measurements of $A_{L L}$ for inclusive jet production extracted from $3.1 \mathrm{pb}^{-1}$ of data at $v_{\mathrm{s}}=200 \mathrm{GeV}$ and $50 \%$ beam polarization. The asymmetry is calculated over the transverse momentum region $5<\mathrm{p}_{\mathrm{T}}<30 \mathrm{GeV} / \mathrm{c}$ and compared with theoretical predictions incorporating several gluon polarization scenarios. Systematic uncertainties from false asymmetries, jet reconstruction, and triggering are discussed. At the present level of statistics, the measured asymmetry disfavors maximal gluon polarization but cannot yet distinguish between other theoretical scenarios.
\end{abstract}

Keywords: pp collisions, RHIC, STAR, $A_{L L}$, jets, longitudinal spin asymmetry

PACS: 13.87.-a, 13.88. + e, 12.38.Qk, 14.20.Dh, 24.70.+s

\section{INTRODUCTION}

The Relativistic Heavy Ion Collider (RHIC) at Brookhaven National Laboratory is the world's first polarized proton collider, providing collisions with a center-of-mass energy of $V_{s}=200 \mathrm{GeV}$ (future running at $V_{\mathrm{s}}=500 \mathrm{GeV}$ is planned)[1]. One of the goals of the RHIC spin program is the measurement of the polarized gluon distribution function, $\triangle \mathrm{G}$. In pQCD, the polarized cross section is a convolution of the polarized quark and gluon distribution functions and the (known) polarized hard partonic scattering cross section. A measurement of the double longitudinal spin asymmetry, $A_{L L}$, in inclusive processes such as jet and pion production in polarized proton collisions would contain contributions from q-g and $\mathrm{g}-\mathrm{g}$ scattering and thus provide sensitivity to $\Delta \mathrm{G}$.

The Solenoidal Tracker at RHIC (STAR) experiment has a near term goal of measuring $\Delta \mathrm{G}$ through inclusive jet and pion measurements. STAR is unique at RHIC in having an acceptance large enough to fully reconstruct jets[3], and recently became the first experiment to release a measurement of $A_{L L}$ in inclusive jet production[2]. Measurements of $A_{L L}$ for inclusive jet production are interesting because they are 
insensitive to fragmentation functions. These proceedings present the first results for $A_{L L}$ for inclusive jet production in STAR's $2005 \mathrm{p}-\mathrm{p}$ data.

\section{ANALYSIS AND RESULTS}

The double longitudinal spin asymmetry $A_{L L}$ for a particular process is defined as

$$
A_{L L}=\frac{1}{P_{1} P_{2}} \frac{\left(N^{++}-R N^{+-}\right)}{\left(N^{++}+R N^{+}\right)}
$$

where $\mathrm{N}^{++}$and $\mathrm{N}^{+-}$refer to the number of jets measured in equal and opposite helicity collisions, $\mathrm{P}_{1}$ and $\mathrm{P}_{2}$ refer to the polarizations of the two beams, and $\mathrm{R}$ refers to the ratio between beam luminosities for equal and opposite beam helicities. In 2003 and 2004, STAR recorded a total of $0.3 \mathrm{pb}^{-1}$ of polarized $\mathrm{p}-\mathrm{p}$ collisions with polarizations ranging from $30-45 \%$. Inclusive jet asymmetry and cross section results from this data have been submitted to PRL[2]. In the 2005 RHIC p-p run, STAR recorded $3.1 \mathrm{pb}^{-1}$ of data, out of which $1.6 \mathrm{pb}^{-1}$ were used for the analysis. Polarizations of both beams were measured once every 3-4 hours using the RHIC CNI Polarimeter; the average polarization was $50 \%$ with a uncertainty of $20 \%$ (relative). Luminosities for both beams were measured using STAR's Beam-Beam Counters (BBCs), azimuthally segmented scintillating detectors located at both ends of the experiment at high pseudorapidity. A typical value for $\mathrm{R}$ was 1.1 ; for the entire run, $\delta \mathrm{R} / \mathrm{R}$ is $\sim 0.001$.

Data were collected with four analysis triggers, each requiring a minimum bias condition (a coincidence between both BBCs) as well as a condition in STAR's Barrel Electromagnetic Calorimeter (BEMC). The BEMC is a lead-scintillator sampling calorimeter with a granularity of $0.05 \times 0.05$ in $\Delta \eta \times \Delta \varphi$ which measures electromagnetic energy deposits in the range $0<\eta<1$ (for the 2005 analysis) and $0<\varphi<2 \pi$. STAR's high tower triggers, HT1 and HT2, sampled events in which any single BEMC tower recorded an energy above 2.8 or $3.8 \mathrm{GeV}$. The jet patch triggers, JP1 and JP2, sampled events in which any of six regions in the BEMC with $\Delta \eta \times \Delta \varphi=1.0 \times 1.0$ recorded a combined energy above 4 or $5.5 \mathrm{GeV}$.

STAR reconstructs jets using a midpoint cone clustering algorithm adapted from the Tevatron[6]. This algorithm combines tower hits in the BEMC and reconstructed tracks from the Time Projection Chamber (TPC)[4]. STAR's TPC sits inside a 0.5 Tesla field that allows the measurement of charged particle transverse momenta up to $20 \mathrm{GeV} / \mathrm{c}$. Only TPC tracks originating from the primary interaction vertex with at least 20 hits and momenta higher than $0.2 \mathrm{GeV}$ were used to reconstruct jets. The jet seed energy was $0.5 \mathrm{GeV}$, and a maximum jet cone radius of 0.4 (in $\eta \times \varphi$ ) was chosen because of the limited BEMC $\eta$ acceptance.

For the asymmetry analysis, only jets responsible for event triggers were used. To minimize detector edge effects, jets whose centroids fell outside the range $0.2<\boldsymbol{\eta}<0.8$ were cut. Finally, backgrounds required the removal of any jet whose summed BEMC energy was more than $80 \%$ of its total energy. Ultimately, roughly 2 million jets were used for the physics analysis, of which 1.4 million came from the JP2 trigger. Only $2 \%$ of the events in this sample contained dijets. 
Figure 1 shows STAR's preliminary 2005 measurement of $A_{L L}$ in inclusive jet production. The theoretical bands on the plot are calculated at NLO and use several possible polarized gluon distribution functions as inputs[5]. The black line, for instance, is taken from an input distribution corresponding to the best fit to DIS data, while the red and green lines correspond to maximally positive and negative gluon polarization scenarios. STAR's data does not yet distinguish between scenarios, aside from ruling out maximally positive gluon polarization. The data are in good agreement with the 2003/2004 results.

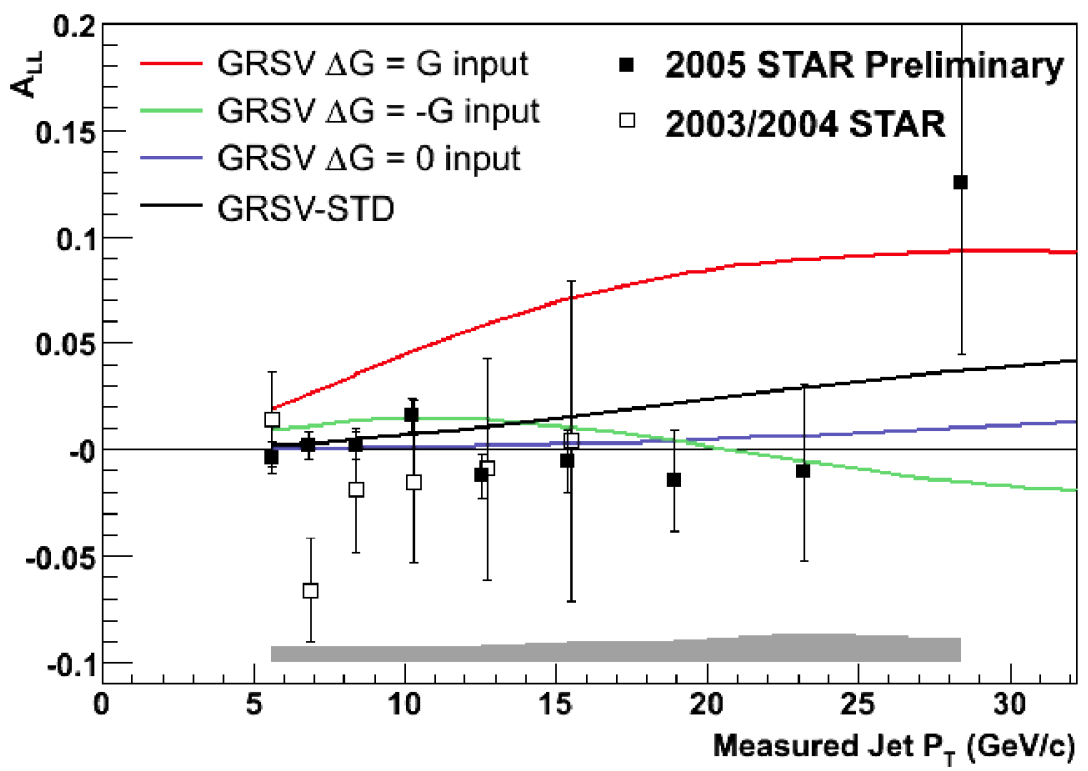

FIGURE 1. STAR's preliminary measurement of the 2005 double longitudinal spin asymmetry for inclusive jet production. Also shown are the results from STAR's 2003/2004 inclusive jet spin analysis. Error bars are purely statistical; the systematic error band for the 2005 data is given at the bottom in gray, and does not include a $25 \%$ scale error from polarization uncertainty. The colored bands show different theoretical predictions for $\Delta \mathrm{G}$ and are discussed in the text.

The systematic error band in Figure 1 is dominated by uncertainties from false asymmetries, trigger bias, and jet reconstruction bias. (Non-longitudinal beam polarization and relative luminosity measurement uncertainties also contributed.) Depending on the cuts used, STAR observed a $1.5-3 \sigma$ (false) single spin asymmetry in one of its beams. The source of this asymmetry is unclear, but a similar false asymmetry in the "like-sign" asymmetry (between the ++ and -- beam helicity states) suggests one anomalous spin state as the culprit. We treat the like-sign asymmetry as a systematic effect and use the dependence between $A_{L L}$ and the like-sign asymmetry to derive a systematic uncertainty.

Trigger bias and jet reconstruction bias were both estimated by combining DIS $\Delta \mathrm{G}$ parameterizations (code donated by Werner Vogelsang) with partonic information from PYTHIA to estimate $A_{L L}$ for each jet, for each detected jet (using GEANT to 
simulate the detector), and for detected events after triggers were applied. The difference between the first two asymmetries was interpreted as a jet reconstruction bias, while the difference between the latter two defined the trigger bias.

\section{SUMMARY AND OUTLOOK}

This contribution gives STAR's preliminary 2005 results for the double spin asymmetry $A_{L L}$ for inclusive jet production in polarized p-p collisions at $\sqrt{s}_{\mathrm{s}}=200 \mathrm{GeV}$. The measured asymmetries are compared to several different predictions for the polarized gluon distribution and disfavor maximal gluon polarization. Data taken in 2006 will significantly increase STAR's figure of merit and should allow STAR to distinguish between the various $\Delta \mathrm{G}$ scenarios.

\section{REFERENCES}

1. G. Bunce, N. Saito, J. Soffer, and W. Vogelsang, Ann.Rev.Nucl.Part.Sci.50,525(2000).

2. B.I. Abelev et al. arXiv:hep-ex/0608030. Submitted to Phys. Rev. Lett.

3. K.H. Ackermann et al., Nucl.Instrum.Meth.A499,624(2003).

4. M. Anderson et al., Nucl.Instrum.Meth.A499,659-678(2003), nucl-ex/0301015.

5. B. Jager, M .Stratmann, and W. Vogelsang, Phys.Rev.D70,034010(2004).

6. G. Blazey et al., hep-ex/0005012. 